\title{
Caracterización y registro del polen atmosférico en la ciudad de Temuco
}

PEDRO MARDONES P.,*****, GUSTAVO DONOSO D.******, ROXANA ROCHA C.****, ALICIA CÓRDOVA J*. y MASUMI GRAU T.*

\section{Characterization and registry of the atmospheric pollen in Temuco, Chile}

The aim of this study was to quantify and to characterize the amount of pollen in the atmosphere of the city of Temuco, Chile from 2006 to 2009. Aerobiological sampling was carried out by using a Seven Day Volumetric Spore-Trap Burkard and the samples were analyzed weekly during the period of study. Results: We determined the 11 principal anemophilus species of urban flora in Temuco responsible for pollinosis. The highest concentration of pollen were present from September till March, being the most important species the Grasses (Gramineae/Poaceae), Cypress (Cupressus sp), and Sorrell (Rumex sp.)

Key words: Pollinosis; pollen; allergic rhinitis; asthma.

\section{Resumen}

Se realizó un estudio con el objetivo de cuantificar y caracterizar la carga polínica en la ciudad de Temuco, entre los años 2006-2009. Para el muestreo aerobiológico se utilizó un captador volumétrico Burkard Seven Day Volumetric Spore-Trap ${ }^{\circledR}$. Las muestras obtenidas fueron analizadas semana tras semanas durante el período en estudio. Con los resultados del conteo polínico semanal se determinaron las 11 principales especies de flora urbana anemófilas de Temuco más susceptibles de producir polinosis. Las mayores concentraciones de polen se obtienen entre Septiembre y Marzo, siendo las especies más importantes: Pastos (Gramineae/Poaceae), Ciprés (Cupressus sp) y Acedera (Rumex sp).

Palabras clave: Polinosis; polen; rinitis alérgica; asma.

\section{Introducción}

Las condiciones geográficas y climáticas de varias ciudades chilenas han favorecido el establecimiento de especies exóticas en desmedro de las nativas, las que se han adaptado y difundido a lo largo de nuestro país. Muchas de estas especies son anemófilas y con antecedentes de inducir alergias $^{1,2,3}$. Esta situación, permite suponer que el reemplazo de la flora nativa ha repercutido en el aumento de la expresión clínica de sensibilización al polen de varias de estas especies, presentes en las ciudades de nuestro país ${ }^{1}$. Esta reacción que provoca el polen, en sujetos susceptibles, es conocida con el nombre de Polinosis. Se trata de una reacción exagerada por parte del sistema inmune ante un alergeno (en este caso polen) que es considerada erróneamente como un elemento dañino ${ }^{2,3}$. Ante esta señal el sistema inmune responde mediante la producción de anticuerpos específicos del tipo IgE iniciando así una serie de eventos inflamatorios en las mucosas respiratorias, que derivan finalmente en los clásicos cuadros de Rinoconjuntivitis Alérgica y en ocasiones Asma Bronquial ${ }^{2,3}$. Los síntomas que ocasiona pueden afectar significativamente la calidad de vida de la personas, llegando a motivar gran número de ausencias laborales. En los niños con síntomas no controlados, puede ocasionar alteraciones del sueño, ausentismo escolar y problemas de aprendizaje.

La presencia de un tipo polínico no es igual a lo largo de los 12 meses del año, dependiendo del ámbito geográfico ${ }^{4-6}$. De esta forma, la diversidad

* Clínica de Enfermedades Respiratorias y Alérgicas Miguel de Servet, Santiago, Chile.

** Escuela de Ciencias Forestales. Universidad Católica de Temuco, Temuco, Chile.

*** Programa Interuniversitario de Doctorado en Ciencias Forestales, Universidad de Concepción.

***** Fundación de Aerobiología y Pólenes. Santiago, Chile. 
y composición del espectro polínico de una ciudad son un reflejo de su paisaje vegetal.

La flora arbórea presente en la ciudad de Temuco tiene sus orígenes de los colonos europeos, además de especies nativas plantadas o circundantes, pastos y malezas provenientes de áreas verdes urbanas, terrenos eriazos y praderas cercanas. En un trabajo realizado por Romero ${ }^{7}$, se determinaron 125 especies arbóreas para la ciudad de Temuco, donde el $75 \%$ son especies introducidas (Europa, Asia y Norte de África). Según este artículo, las familias más representadas fueron Rosaceae (17\%), Betulaceae (9,5\%), Fabaceae (9\%), Proteaceae (8,6\%), nombrando como especies más representativas a: Betula pendula, Malus baccata, Robinia pseudoacacia, Embothrium coccineum y Maytenus boaria.

La ciudad de Temuco está ubicada en la zona centro- sur del país, a $670 \mathrm{~km}$ de Santiago y a una elevación de 114 metros sobre el nivel del mar. Predomina un clima templado lluvioso con influencia mediterránea, cuya característica principal es que las precipitaciones se hacen presentes en todos los meses del año, concentrándose principalmente en el período invernal, siendo enero y febrero los meses más secos. El régimen térmico no sufre mayores alteraciones que las debidas a su distancia del mar? $^{7}$.

De lo anterior podemos desprender que la dinámica de los registros atmosféricos de polen variarán dependiendo de las distintas especies y condiciones climáticas ${ }^{4,8,9}$, dando lugar a su característica más importante y distintiva, su estacionalidad.

En Chile, los trabajos orientados a describir cargas polínicas atmosféricas han sido escasos. El primer intento de investigar y medir el polen atmosférico fue realizado por el grupo de Hoffman, Riveros, Araya, Rivera, Avila et $\mathrm{al}^{2}$. Este trabajo, se llevó a cabo con un captador gravimétrico, lo que limitaba sus conclusiones al definir cualitativamente el polen presente y su estacionalidad. Además, fue insuficiente para definir cuantitativamente las concentraciones polínicas. También existen algunas aproximaciones en tesis de grado, como la de Blanc en $1942^{3}$.

Posteriormente Rojas, Roure, Galleguillos y Mardones ${ }^{1}$ determinaron mediante métodos volumétricos la lluvia polínica de Santiago durante tres años. Ambos trabajos establecieron las bases racionales para la implementación de las baterías de alergenos para el diagnóstico de los cuadros de alergia respiratoria en la ciudad de Santiago.

Conocer las cargas de polen ambiental es básico para el diagnóstico, tratamiento y prevención de las polinosis, permitiéndonos determinar la diversidad polínica, época de presencia y períodos de mayor concentración de cada polen ${ }^{10}$.

El presente trabajo, describe la concentración de polen ambiental por especies susceptibles de causar polinosis en los habitantes de la ciudad de Temuco.

\section{Metodología}

La estación designada para el monitoreo polínico de la ciudad de Temuco fue ubicada en el campus San Francisco de la Universidad Católica de Temuco, según las guías sobre la colocación de los colectores publicadas por Ogden et $\mathrm{al}^{11}$.

El estudio de la lluvia polínica de la ciudad de Temuco se llevó a cabo mediante un captador volumétrico de polen y esporas Burkard Seven Day Volumetric Spore-Trap ${ }^{\circledR}$, inspirado íntegramente en el método desarrollado por Hirst ${ }^{5,9}$ en 1952. Consiste en una cámara de admisión de aire con un débito de $10 \mathrm{~L} / \mathrm{min}$, que hace pasar el flujo a través de una hendidura de 14 × $2 \mathrm{~mm}$, que está siempre frente a la dirección del viento gracias a que el colector va montado sobre una veleta. La fuente de aire aspirada se enfrenta sobre una cinta vaselinada de $345 \mathrm{~mm}$ que cubre un tambor rotatorio, que se desplaza frente a la hendidura a una velocidad de $2 \mathrm{~mm} / \mathrm{h}$, produciéndose la impactación de los granos polínicos sobre la cinta durante un ciclo de 7 días.

El período de muestreo y conteo de pólenes se efectuó entre Mayo 2006 a Abril 2009, realizándose recolecciones diarias, las que fueron llevadas al laboratorio para su conteo. Para la determinación de las concentraciones diarias de polen se realizó un conteo de las muestras obtenidas, observando un campo visual de 400X de un microscopio óptico Nikon E200 ${ }^{\circledR 12}$. Tras estos conteos se identifican y cuantifican los granos promedios de polen presentes por volumen de aire expresados en granos de polen por metro cúbico de aire.

Con los promedios diarios se calcularon promedios semanales para cada especie, los que fueron tabulados a lo largo de todo el período en estudio, obteniéndose promedios generales para cada una de las especies polínicas. Posteriormente, se escogieron las especies de mayor importancia, según concentración y antecedentes de poder alergizante, descrito en la literatura, ${ }^{1,13,14}$. Tanto la operación del captador como la conversión e interpretación de las muestras obtenidas de este, se hicieron de acuerdo a las recomendaciones dadas por la World Allergy Organization (WAO) ${ }^{15}$. 


\section{Resultados}

Los registros de polen atmosféricos determinaron para la ciudad de Temuco la presencia de 11 especies principales, sospechosas de causar alergia polínica o polinosis (Tabla 1).

Los promedios semanales de los tres años de estudio fueron tabulados y promediados entre sí para cada especie polínica, arrojando un total y promedio anual de los tres años para cada una de ellas, sobre la base de lo cual se estableció que las más representativas fueron: Gramineas (Gramineae/Poaceae) con un promedio anual 4.282,6 granos de polen $/ \mathrm{m}^{3}$ de aire, Ciprés (Cupressus sp) con 1.943,9 granos de polen $/ \mathrm{m}^{3}$ y Acedera (Rumex sp) con 1.165,6 granos de polen $/ \mathrm{m}^{3} \mathrm{de}$ aire (Tabla 2).

Según los registros, cada especie comenzó su período de polinización de forma diferente. De tal manera, podemos decir que la primera especie en presentarse fue el Ciprés en la tercera semana de Junio, luego Olmo y Aliso en la tercera de Julio. Durante Septiembre, mes en el que comienzan a polinizar 5 de las 11 especies, lo hicieron el Roble, el Abedul, Plátano, Arce y los Pastos de manera muy solapada entre las $1^{\mathrm{a}}, 2^{\mathrm{a}}$ y $3^{\mathrm{a}}$ semana,

Tabla 1. Tipos polínicos seleccionadas para la ciudad de Temuco, Chile

\begin{tabular}{|lll|}
\hline \multirow{3}{*}{ Árboles } & Nombre común & Nombre científico \\
& Aliso & Alnus glutinosa (L.) Gaertn \\
& Arce & Acer sp \\
& Abedul & Betuna pendula Roth \\
& Ciprés & Cupressus sp \\
& Olmo & Ulmus sp \\
& Plátano oriental & Platanus x acerifolia (Ait.) Willd. \\
& Roble & Nothofagus tipo oblicua (Mirb.) Oerst \\
& Mirtáceas [Arrayán] & Myrtaceae \\
Pastos & Pastos & Gramineae/Poaceae \\
Malezas & Acedera & Rumex sp \\
& Llantén & Plantaginaceae \\
\hline
\end{tabular}

Tabla 2. Concentración promedio, período de polinización y peak de las especies registradas

\begin{tabular}{|c|c|c|c|c|}
\hline & $\begin{array}{c}\text { Total anual } \\
\text { promedio tres años } \\
\text { (granos de polen } / \mathbf{m}^{3} \text { ) }\end{array}$ & $\begin{array}{l}\text { Período de } \\
\text { polinización }\end{array}$ & $\begin{array}{c}\text { Promedio semana } \\
\text { peak } \\
\text { (granos de polen } / \mathbf{m}^{3} \text { ) }\end{array}$ & Semana peak \\
\hline Arce & 294,4 & Septiembre-noviembre & 9,8 & $3^{\text {a }}$ Octubre \\
\hline Aliso & 278,6 & Julio-agosto & 20,7 & $1^{\mathrm{a}}$ Agosto \\
\hline Abedul & 501,7 & Septiembre-octubre & 19,5 & $4^{\text {a }}$ Septiembre \\
\hline Ciprés & 1943,9 & Junio-noviembre & 25,8 & $2^{\text {a }}$ Octubre \\
\hline Mirtáceas & 707,9 & Octubre-noviembre & 16,3 & $4^{\mathrm{a}}$ Octubre \\
\hline Roble & 840,9 & Septiembre-octubre & 25,6 & $4^{\text {a }}$ Septiembre \\
\hline Olmo & 352,9 & Julio-septiembre & 18,6 & $2^{\mathrm{a}}$ Agosto \\
\hline Plátano & 353,6 & Septiembre-octubre & 19,4 & $1^{\text {a }}$ Octubre \\
\hline Plántago & 516,4 & Noviembre-febrero & 8,3 & 2a Diciembre \\
\hline Acedera & $1.165,6$ & Octubre-febrero & 22,6 & $5^{\text {a }}$ Noviembre \\
\hline Pastos & $4.282,6$ & Septiembre-marzo & 146,1 & $1^{\mathrm{a}}$ Enero \\
\hline
\end{tabular}


Tabla 3. Presencia atmosférica de los pólenes seleccionados en relación a la concentración total anual

\begin{tabular}{|c|c|c|}
\hline & $\begin{array}{l}\text { Total anual promedio tres años } \\
\text { (granos de polen } / \mathbf{m}^{3} \text { ) }\end{array}$ & $\begin{array}{c}\text { Presencia } \\
\text { atmosférica (\%) }\end{array}$ \\
\hline Arce & 294,4 & 2,6 \\
\hline Aliso & 278,6 & 2,5 \\
\hline Abedul & 501,7 & 4,5 \\
\hline Ciprés & $1.943,9$ & 17.3 \\
\hline Mirtáceas & 707,9 & 6,3 \\
\hline Robles nativos & 840,9 & 7.5 \\
\hline Olmo & 352,9 & 3,1 \\
\hline Plátano & 353,6 & 3,1 \\
\hline Plántago & 516,4 & 4,6 \\
\hline Acedera & $1.165,6$ & 10,4 \\
\hline Pastos & $4.282,6$ & 38,1 \\
\hline Total & $11.238,5$ & 100 \\
\hline
\end{tabular}

respectivamente. Las Mirtáceas/Acedera lo hicieron la $2^{\text {a }}$ semana de Octubre, mientras que el último en polinizar fue el Plántago en la $3^{\mathrm{a}}$ semana de Noviembre (Tabla 2).

La presencia atmosférica promedio de cada especie, en relación a la concentración promedio anual de todos los pólenes se muestra en la Tabla 3.

La distribución estacional de los pólenes, obtenida de los promedios semanales de cada año para cada una de las especies, en el período de estudio, se muestra en forma independiente para cada especie en las Figuras (1 a 11).

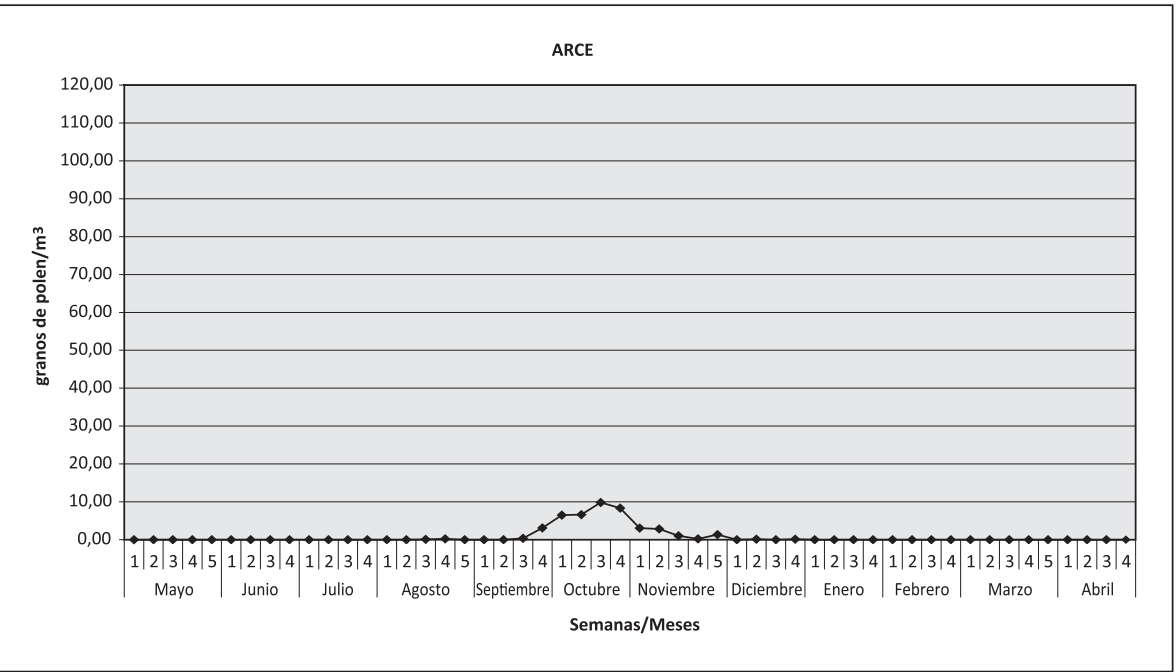

Figura 1. Recuento de pólenes de Arce en la ciudad de Temuco (2006-2009).

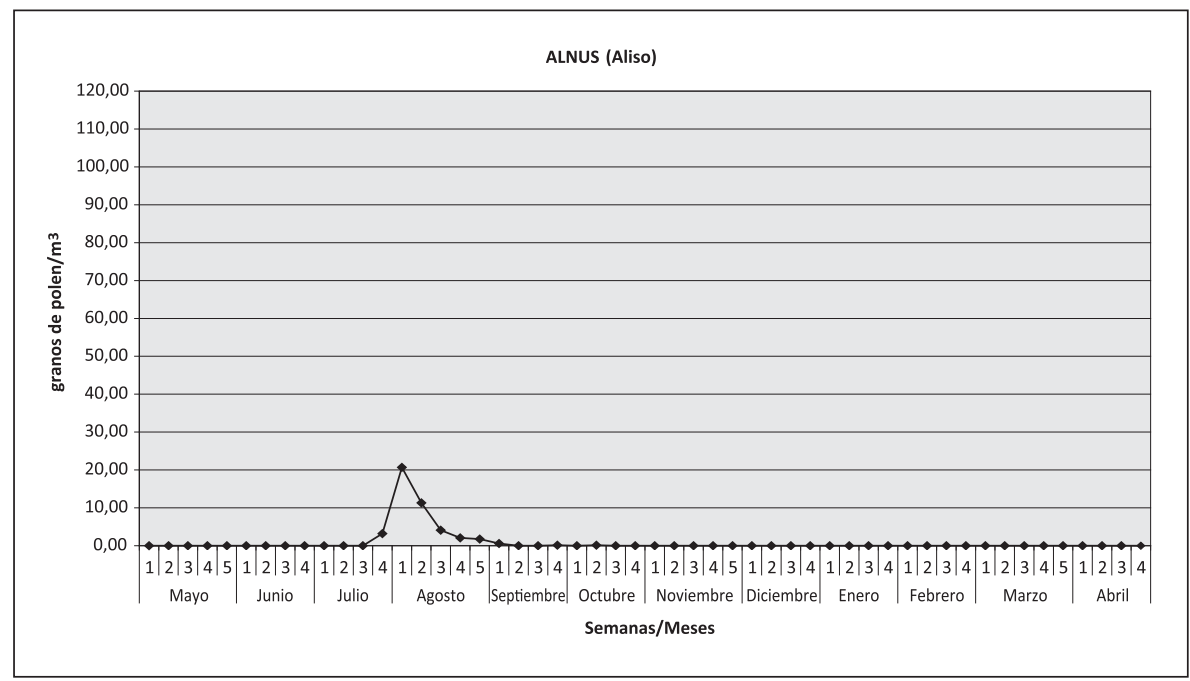

Figura 2. Recuento de pólenes de Aliso en la ciudad de Temuco (2006-2009). 

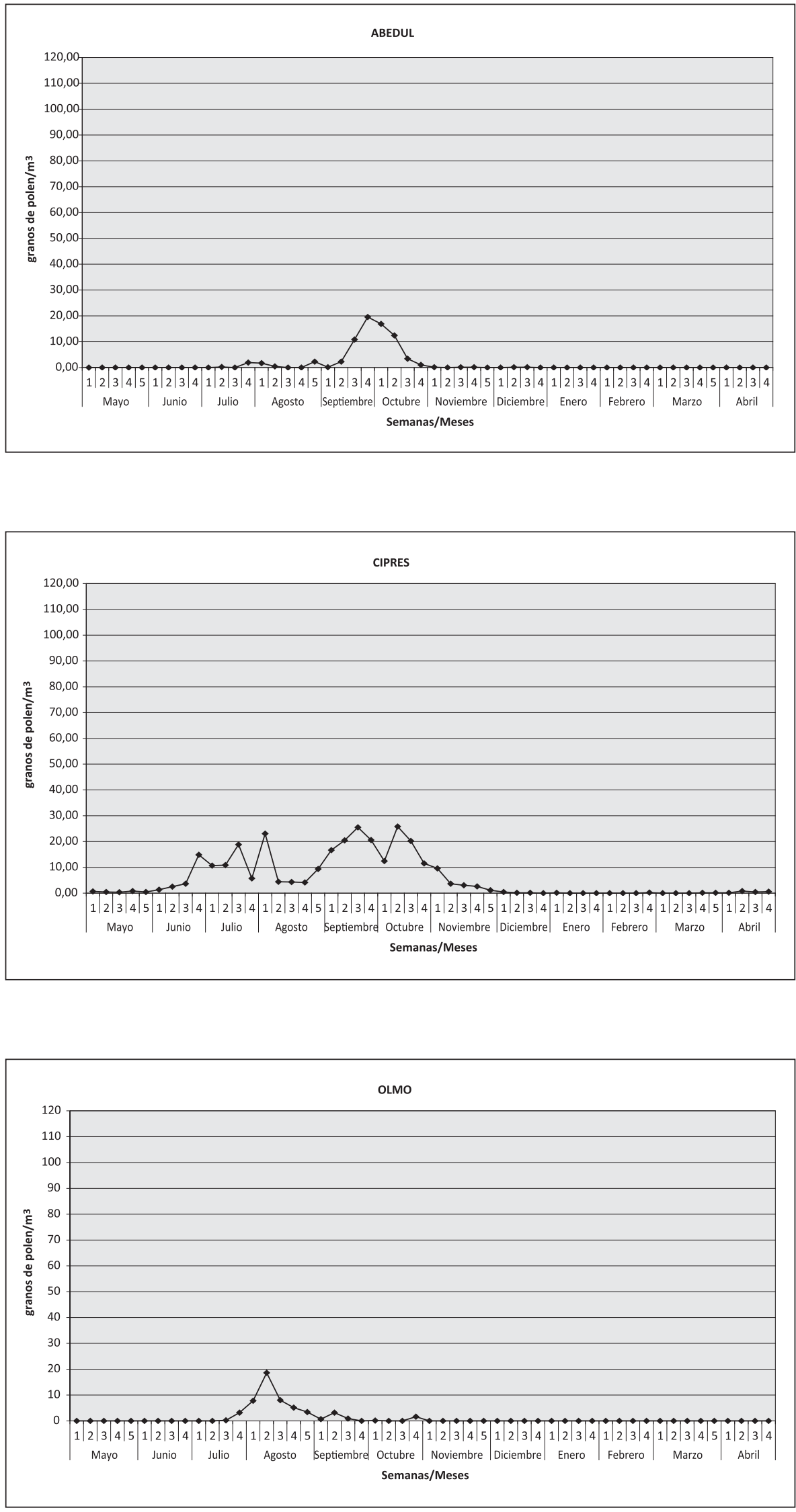

Rev Chil Enf Respir 2011; 27: 7-15
Figura 3. Recuento de pólenes de Abedul en la ciudad de Temuco (2006-2009).

Figura 4. Recuento de pólenes de Ciprés en la ciudad de Temuco (2006-2009).

Figura 5. Recuento de pólenes de Olmo en la ciudad de Temuco (2006-2009). 

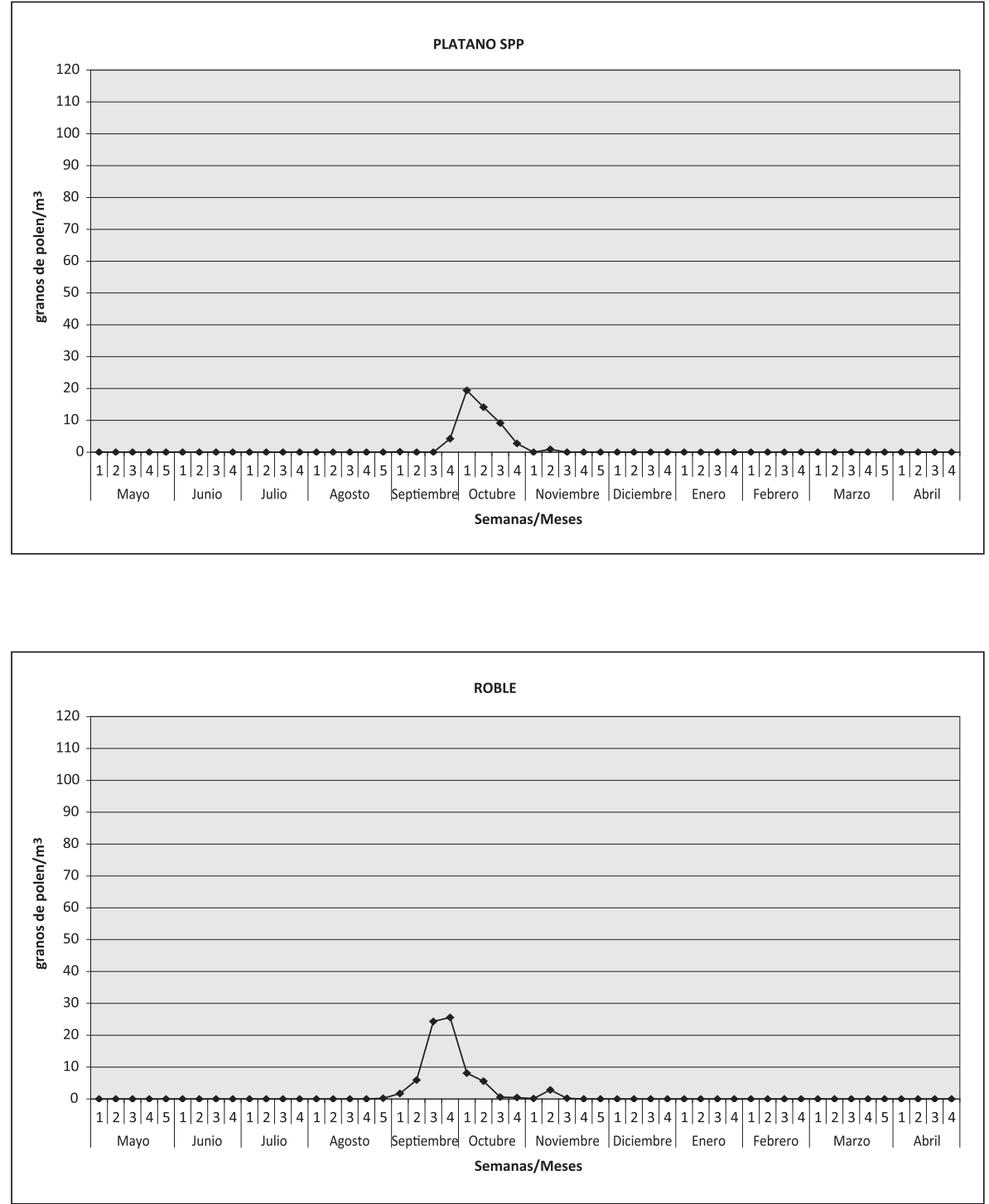

Figura 6. Recuento de pólenes de Plátano spp en la ciudad de Temuco (2006-2009).

Figura 7. Recuento de pólenes de Roble en la ciudad de Temuco (2006-2009).

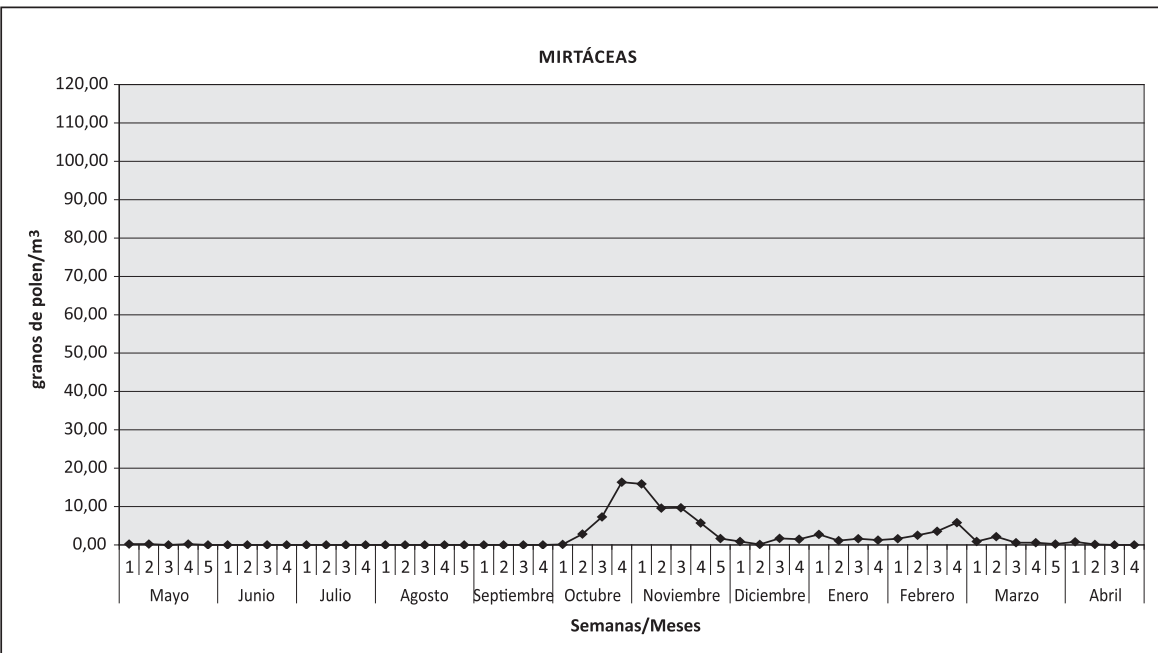

Figura 8. Recuento de pólenes de Mirtáceas spp en la ciudad de Temuco (2006-2009). 


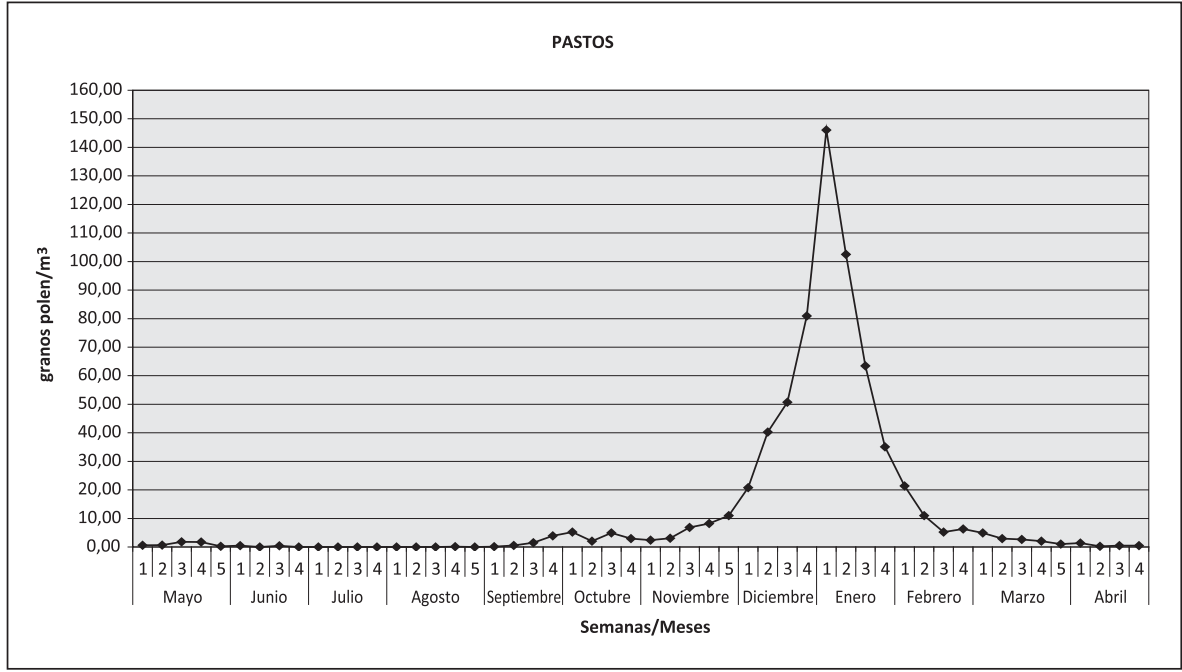

Figura 9. Recuento de pólenes de Pastos (Gramíneas) en la ciudad de Temuco (20062009).

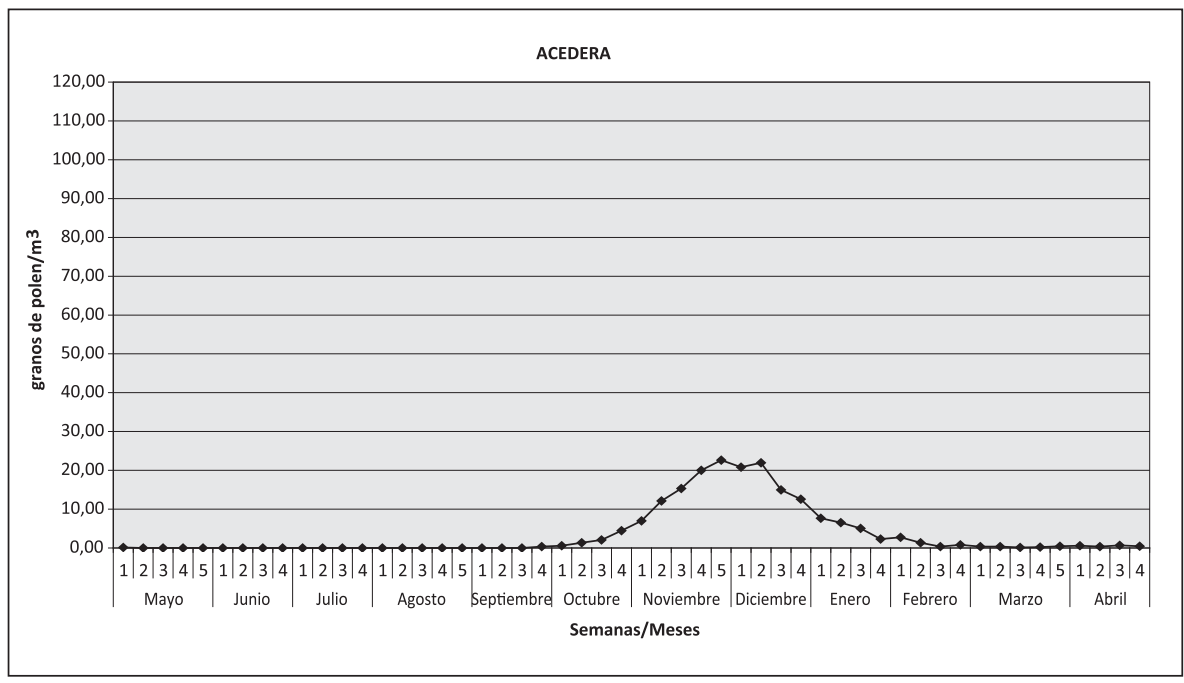

Figura 10. Recuento de pólenes de Acedera en la ciudad de Temuco (2006-2009).

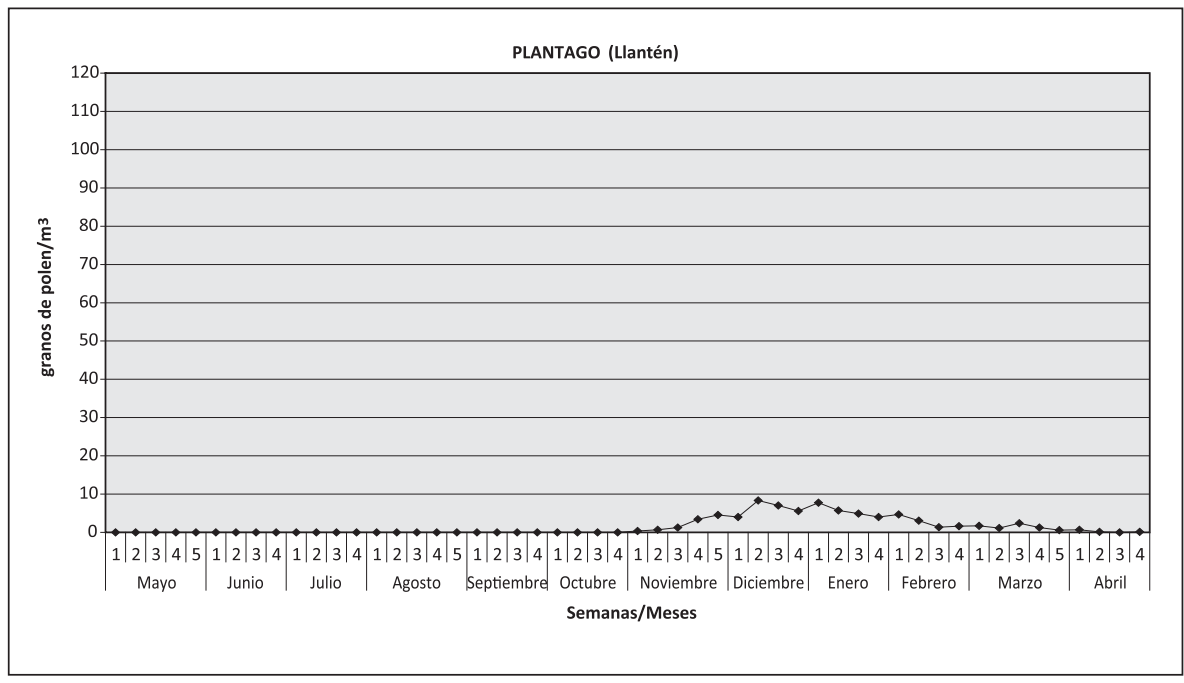

Figura 11. Recuento de pólenes de Llantén en la ciudad de Temuco (2006-2009). 


\section{Discusión}

La ciudad de Temuco es una ciudad con una variada flora urbana lo que se refleja en la diversidad de taxas polínicas registradas. Sin embargo, desde el punto de vista de las posibilidades de estos pólenes de generar alergia respiratoria, se seleccionaron once especies capaces de sensibilizar el tracto respiratorio, ya sea por su potencial alergénico reconocido en la literatura, en el caso de las exóticas o cosmopolitas, o por la concentración atmosférica, como el caso de algunas nativas que se exponen en este artículo.

Se detectan, entre las especies de pólenes arbóreos registradas, seis exóticas (Arce, Aliso, Abedul, Ciprés, Olmo y Plátano), dos nativas (Roble y Mirtáceas spp [Ej: Arrayán]), Gramíneas o pastos, sin especificar diferencias a nivel polínico de las diferentes especies bajo microscopía óptica, y malezas cosmopolitas (Pastos, Acedera [acelga, vinagrillo] y Llantén). Existen múltiples antecedentes en trabajos realizados en el mundo donde se muestra una alta sensibilidad a las especies exóticas y hierbas cosmopolitas encontradas en Temuco. Destacamos los artículos de Paternelli y cols, Zagreb, Croacia ${ }^{16}$, de Subiza en España ${ }^{17}$, de Boralli et al en India ${ }^{18}$ y D'Amato en Italia $^{19}$, donde detectan como uno de los principales alergenos en sus respectivos estudios a varios de los pólenes registrados en la atmósfera de Temuco, entre ellos el Abedul y otras betuláceas, el Plátano de sombra, el Ciprés, Gramíneas del tipo poaceas, el Plantago y la Acedera.

La estacionalidad está muy marcada por la presencia abundante de pólenes de pastos durante los meses de Diciembre, Enero y Febrero. Además, entre los meses de Agosto a Noviembre polinizan árboles exóticos de reconocido poder alergénico como son el Ciprés, Abedul y Olmo, tal como lo describen Patemeli y Subiza, entre otros. Las concentraciones aunque no muy altas, son capaces de generar síntomas en algunos individuos muy sensibles, o por exposiciones individuales mayores, como sería el tema de los abedules plantados abundantemente en la zona residencial de Temuco.

Por otra parte el plátano oriental y el arce, árboles muy prevalentes en otras ciudades de Chile, como Santiago y Talca, y claramente alergénicos, se registran en concentraciones bajas en Temuco. En la ciudad de Santiago el plátano oriental es responsable del $35 \%$ de la polinosis entre los meses de Agosto a Septiembre ${ }^{1}$ y en Talca su prevalencia también es alta aunque no hay estudios previos que correlacionen los niveles de pólenes con los síntomas de los pacientes.
Junto a los árboles recién mencionados, los pólenes nativos de Mirtáceas (pataguas, arrayanes) y robles contribuyen a la carga alergénica entre los meses de Septiembre y Noviembre y estos deberían estudiarse en relación al impacto en la sensibilización de los pacientes con Rinocojuntivitis Alérgicas (RCA) y/o Asma en Temuco. No hay estudios sobre el poder alergizante de los árboles nativos.

Por otra parte, el resto de los pólenes se encuentra en niveles en que el riesgo de alergia no es tan importante, pero que podría llegar a serlo en caso de que se comience a sobrecultivar alguna especie en particular, como es el caso del abedul en algunas zonas de la ciudad.

En resumen, Temuco registra una estacionalidad marcada en cuanto al registro de pólenes, y varios de ellos tienen antecedentes de alergia importante ${ }^{16-19}$. De todos los pólenes encontrados, los de pastos son los más frecuentes e importantes desde el punto de vista alergénico y entre los árboles, tanto el abedul como el ciprés son ampliamente reconocidos como alergizantes y presentan niveles significativos que podrían generar síntomas en pacientes sensibilizados.

En cuanto a los nativos, es importante destacar las mirtáceas y roble, los cuales por carecer de antecedentes es necesario estudiar la repercusión clínica que tienen en la población.

Habiendo establecido las características principales de la "lluvia polínica" en la ciudad de Temuco es muy importante en esta etapa estudiar, mediante la realización de test cutáneos, y de test de provocación conjuntival y bronquial, cómo estos pólenes inciden en los síntomas de RCA y/o Asma en la población de Temuco, lo que implicaría un trabajo en pacientes Riniticos y/o Asmáticos donde se debiera evaluar, con extractos alergénicos, la sensibilidad a los diferentes pólenes registrados en la atmósfera mediante test cutáneos. Este procedimiento podría proporcionar información respecto a los alergenos relevantes de esta ciudad que deberían ser incluidos siempre en los test de alergia para los pacientes.

Actualmente esta información no existe y se utilizan protocolos extraídos de la literatura extranjera o de otras ciudades como Santiago. Es muy probable que con esta información se puedan ahorrar muchos recursos, ya que se racionalizaría y optimizarían los gastos en extractos alergénicos para tests basados en la información local.

Por otra parte, nos parece importante la labor de monitoreo y vigilancia aerobiológica continua en la ciudad de Temuco. Mediante esta acción, se podría obtener información relevante para 
planificar la forestación urbana de una manera sustentable, que no hipoteque la calidad de vida de quienes habitan la ciudad. Además, el entregar una información oportuna a la comunidad respecto a los niveles de pólenes alergizantes, mejora mucho la oportunidad y precisión en el diagnóstico y tratamiento de estas patologías, logrando así el objetivo final de mejorar la calidad de vida de los pacientes.

\section{Agradecimientos}

Los autores agradecen el apoyo para este artículo del Proyecto FONIS, código SA07I20058. Además, al Laboratorio Merck Sharp \& Dohme Chile.

\section{Bibliografía}

1.- ROJAS G, ROURE J, GALLEGUILLOS F, MARDONES P. Aeropalinología de Santiago. Rev Chil Enf Respir 1999; 15: 141-55.

2.- HOFFMAN A V, RIVEROS F, ARAYA S, RIVERA O, AVILA G, MONTENEGRO G, et al. Identificación y recuento del pólen alergénico atmosférico en el Centro de Santiago (1976-1971). Rev Med Chile 1978; 106: 595-600.

3.- BLANC H. Relaciones clínicas y dermatológicas de la polinosis. Tesis de Médico. Imprenta el Chileno 1942.

4.- VALERO A, PICADO C. Polinosis. Servicio de Neumología y Alergia Respiratoria ICPCT. Hospital Clínic. Barcelona, 2002; 17-21.

5.- SUBIZA J, JEREZ M, JIMÉNEZ J A, NARGANES M, CABRERA M, VARELA S, et al. Allergenic Pollen and Pollinosis in Madrid. J Allergy Clin Immunol 1995; 96: 15-23.

6.- GUTIÉRREZ M, SABARIEGO S, CERVIGÓN P. Calendario Polínico de Madrid (Ciudad Universitaria). Período 1994-2004. Lazaroa 2006; 27: 21-7.

7.- ROMERO-MIERES M, REBOLLEDO S, JARAMILLO P. Árboles ornamentales de la ciudad de Temuco,
Región de la Araucanía (IX), Chile. Chloris Chilensis, 2009; $12: 1$.

8.- SUBIZA J, BRITO F, OLA J, MORAL A, FERNÁNDEZ J, JEREZ M, et al. Pólenes Alergénicos y Polinosis en 12 Ciudades Españolas. Revista Española de Alergia e Inmunología Clínica 1998; 13: 45-8.

9.- SPIEKSMA F. Regional European Pollen Calendars. En: D’ Amato, G. 1991. Allergenic polllen and pollinosis in Europe. Blackwell Scientific Publication. Oxford. UK. 1991.

10.- GARCÍA GONZÁLEZ J. Polinosis, una enfermedad en aumento. Revista Española de Alergía 1999; 5: 13-6.

11.- OGDEN, RAYNOR G, HAYES J, LEWIS D, HAINES J. Manual for sampling airborne pollen. Nueva York; Hafenr press, 1974.

12.- DOMÍNGUEZ E, GALÁN C, VOLLAMANDOS F, INFANTE F. Handling and evaluation of the data from the aerobiological sampling. Rea Monogr 1992; 1: 1-18.

13.- LIEBERS V, SANDER I, VAN DAMPEN V, RAULFHEIMSOTH M, ROZYNEK P, BAUR X. Overview on denominated allergens. Clin Exp Allergy 1996; 26: 494-516.

14.- SUBIZA E, SUBIZA J, JEREZ M. Aerobiología de las gramíneas en los climas de España. Rev Esp Alergol Inmunol Clin 1989; 4: 45-50.

15.- HASNAIN S, KATELARIS C, NEWBEGIN E, SINGH A. Aeroallergen Monitoring Standard for the Asia Pacific Region a Wao Manual for the use of the Burkard Volumetric Spore Trap and Burkard Personal Volumetric Air Sampler. World Allergy Organization 2003.

16.- PETERNEL R, MILANOVIC S M, HRGA I, MILETA T, CULIG J Incidence of Betulaceae Pollen and Pollinosis in Zagreb, Croatia, 2002-2005. Ann Agric Environ Med 2007; 14: 87-91.

17.- SUBIZA J. Pólenes alergénicos en España. Allergol et Immunopathol 2004; 32: 121-4.

18.- BORAL D, CHATTERJEE S, BHATTACHARYA K. The Occurrence and Allergising Potential of Airborne Pollen in West Bengal, India. Ann Agric Environ Med 2004; 11: 45-52.

19.- D`AMATO G, LOBEFALO G, Allergenic pollens in the southern Mediterranean area, J Allergy Clin Immunol 1989; 83: 116-22.

Correspondencia a:

Dr. Pedro Mardones Precht

Clínica Miguel de Servet

Almirante Pastene \# 150

Providencia. Santiago, Chile.

Fono: 6406546 - 7216612

E-mail: pemardo@gmail.com 